\title{
High Luminescence White LEDs Prepared with 2D Island-Pattern of Quantum Dots Dispersed Photopolymer Films
}

\author{
Hyun-Guk Hong, ${ }^{1}$ Min-Ho Shin, ${ }^{1}$ Hyo-Jun Kim, ${ }^{1}$ Jinsoo Shin, ${ }^{1,2}$ and Young-Joo Kim ${ }^{1}$ \\ ${ }^{1}$ Department of Mechanical Engineering, Yonsei University, Seoul 120-749, Republic of Korea \\ ${ }^{2}$ LCD ReD Team, Samsung Display Co., Ltd., Asan 336-746, Republic of Korea
}

Correspondence should be addressed to Young-Joo Kim; yjkim40@yonsei.ac.kr

Received 20 February 2015; Accepted 24 May 2015

Academic Editor: Hsueh-Shih Chen

Copyright (c) 2015 Hyun-Guk Hong et al. This is an open access article distributed under the Creative Commons Attribution License, which permits unrestricted use, distribution, and reproduction in any medium, provided the original work is properly cited.

\begin{abstract}
Since the reabsorption loss among different size quantum dots (QDs) is a critical issue in the QD based white LEDs, we proposed and fabricated new film structure of 2D island-patterns consisting of separate green and red QDs dispersed photopolymer patterns in a zigzag form. A small air-gap such as $60 \mu \mathrm{m}$ between QD islands helps to control the optical path at the interface to reduce the lateral reabsorption loss to enhance the optical efficiency of white LED. The 2D island-patterns of QD phosphor film were fabricated using a UV imprinting process and compared the optical efficiency with the other QD film structure prepared with same QD concentrations and thicknesses such as a mixed and separately layered QD structure. Experimental and simulation analysis were performed to confirm the better optical efficiency from the 2D island-patterns of QD films due to the reduced reabsorption loss. High luminescence white LED was finally realized with 2D island-patterns of QD film, resulting in a luminous efficiency of $62.2 \mathrm{~lm} / \mathrm{W}$ and CRI of 83 with CCT of $4537 \mathrm{~K}$ at the operation current of $60 \mathrm{~mA}$.
\end{abstract}

\section{Introduction}

Semiconductor quantum dots (QDs) have recently received an attention to be used in the field of optoelectronics and displays due to their color converting property based on the quantum confinement effect. For instance, it is possible to tune the emission wavelength of QDs simply by varying their size in the nanometer range. Some advantages such as high quantum efficiency, broad range of emission wavelengths, and good photostability make QDs preferable luminescent nanomaterials in many applications [1-5]. For example, the fabrication of QD based white LEDs involves the use of a blue LED chip as blue light and excitation energy source, along with green and red QDs to produce white light $[6,7]$. However, the optical performance is affected by the reabsorption among QDs if the different size QDs are blended together. A reabsorption loss at red QDs with the light emitted from green QDs occurs due to their overlapped range of absorption and emission wavelengths. This imposes an upper limit of color conversion efficiency, making it difficult to achieve high luminescence white LEDs using QD nanomaterials. Some research papers reported the fabrication of noble structure to improve the quantum efficiency in the QD film, including the stacking of different size QDs in separate layers $[8,9]$. However, this separately layered QD structure still includes the possibility of reabsorption loss to the vertical direction of QD layers.

To overcome this reabsorption issue in the phosphor film based on QD dispersed polymer, we proposed 2D islandpattern of QD films, in which the green and red QDs are arranged separately in a zigzag form on the same layer with an air-gap between QD islands. The effect of air-gap in newly proposed QD film structure was evaluated by an optical simulation. Then we fabricated the 2D island-pattern of QD film using a UV imprinting process. For the comparison, two other QD film structures such as the mixed and separately layered QD structures were prepared and evaluated. Then the color conversion efficiency from different QD films was measured to understand the difference of reabsorption loss. Finally high luminescence white LED was fabricated with 2D island-pattern of QD dispersed photopolymer film to confirm the enhanced optical efficiency with 2D island-patterns. 

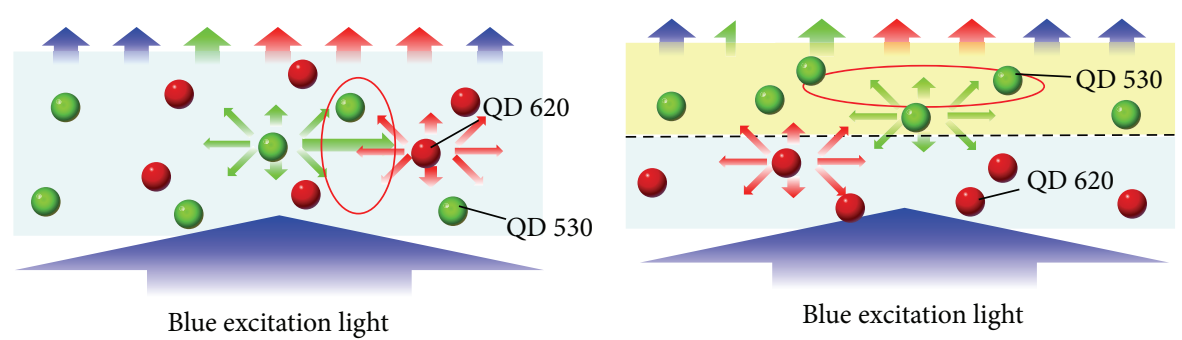

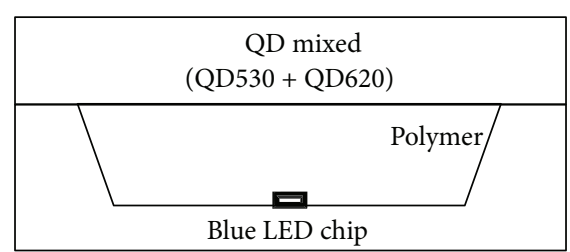

(a)

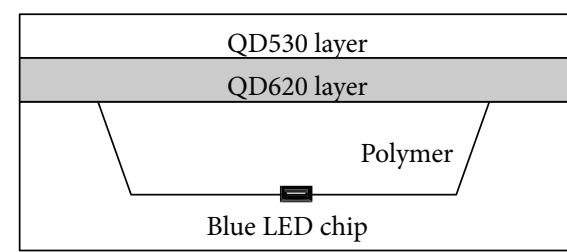

(b)
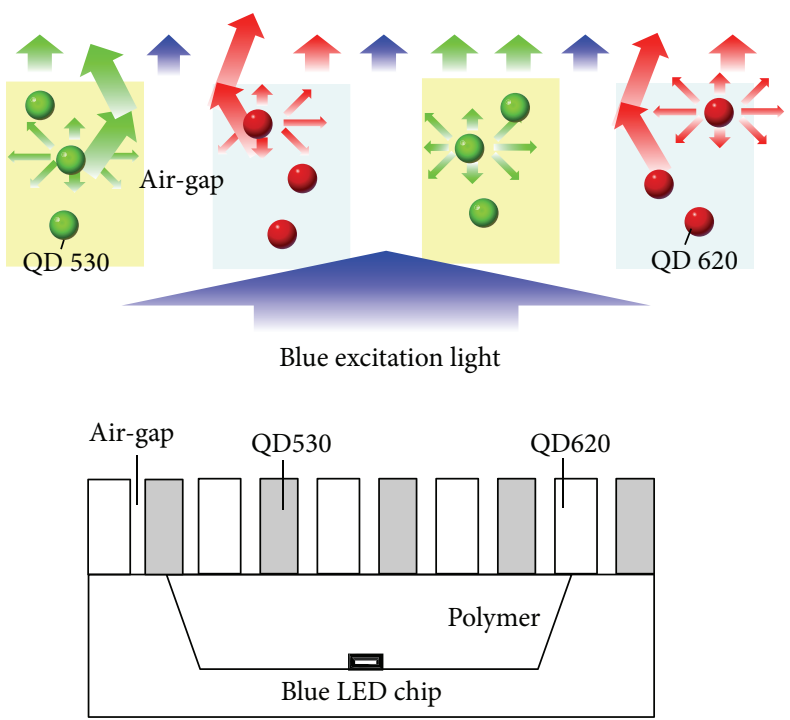

(c)

FIGURE 1: Schematic diagram for the color conversion and reabsorption issue in different types of QD film for white LEDs: (a) mixed, (b) separately layered, and (c) 2D island-patterns of QD dispersed photopolymer films.

\section{Design and Fabrication Methods}

2.1. Proposal of QD Film with 2D Island-Patterns. The QD based white LEDs can be achieved by receiving higher energy from a blue LED chip and converting to longer wavelengths of green and red lights. A remote type phosphor film was considered in this study to improve thermal and photonic stabilities of QDs [10]. Three QD film structures were considered in this study, including (1) mixed, (2) separately layered, and (3) newly proposed 2D island-patterns of QD films, as shown in Figure 1. A mixed QD structure can be prepared by simply mixing two different size QDs having $530 \mathrm{~nm}$ and $620 \mathrm{~nm}$ emission peaks. A separately layered QD structure is fabricated by locating two QDs into different layers. The structure of 2D island-patterns of QD film involves the lateral alignment of two different size QDs in a zigzag form on the same layer. In a mixed type, both QDs are simply blended together, resulting in severe reabsorption by red $620 \mathrm{~nm}$ QDs for the light emitted from green $530 \mathrm{~nm}$ QDs. In case of separately layered type, red QDs are placed in lower layer to decrease the reabsorption loss, but there is still a reabsorption loss since the green light converted from $530 \mathrm{~nm}$ QDs is emitted to all directions to be reabsorbed in $620 \mathrm{~nm}$ QDs.

Thus we proposed and designed new 2D island-patterns of QD film, which is designed to control the location and distance between green and red QDs island-patterns, thereby decreasing the reabsorption loss. In addition, a small airgap between QD islands can control the optical path by total internal reflection at the interface to reduce the lateral reabsorption loss, resulting in the improvement of optical efficiency.

2.2. Fabrication and Evaluation of $Q D$ Films. For the $\mathrm{QD}$ phosphor films, the $530 \mathrm{~nm}$ green and $620 \mathrm{~nm}$ red QDs having a CdSe/ZnS core-shell structure were made by ourselves 


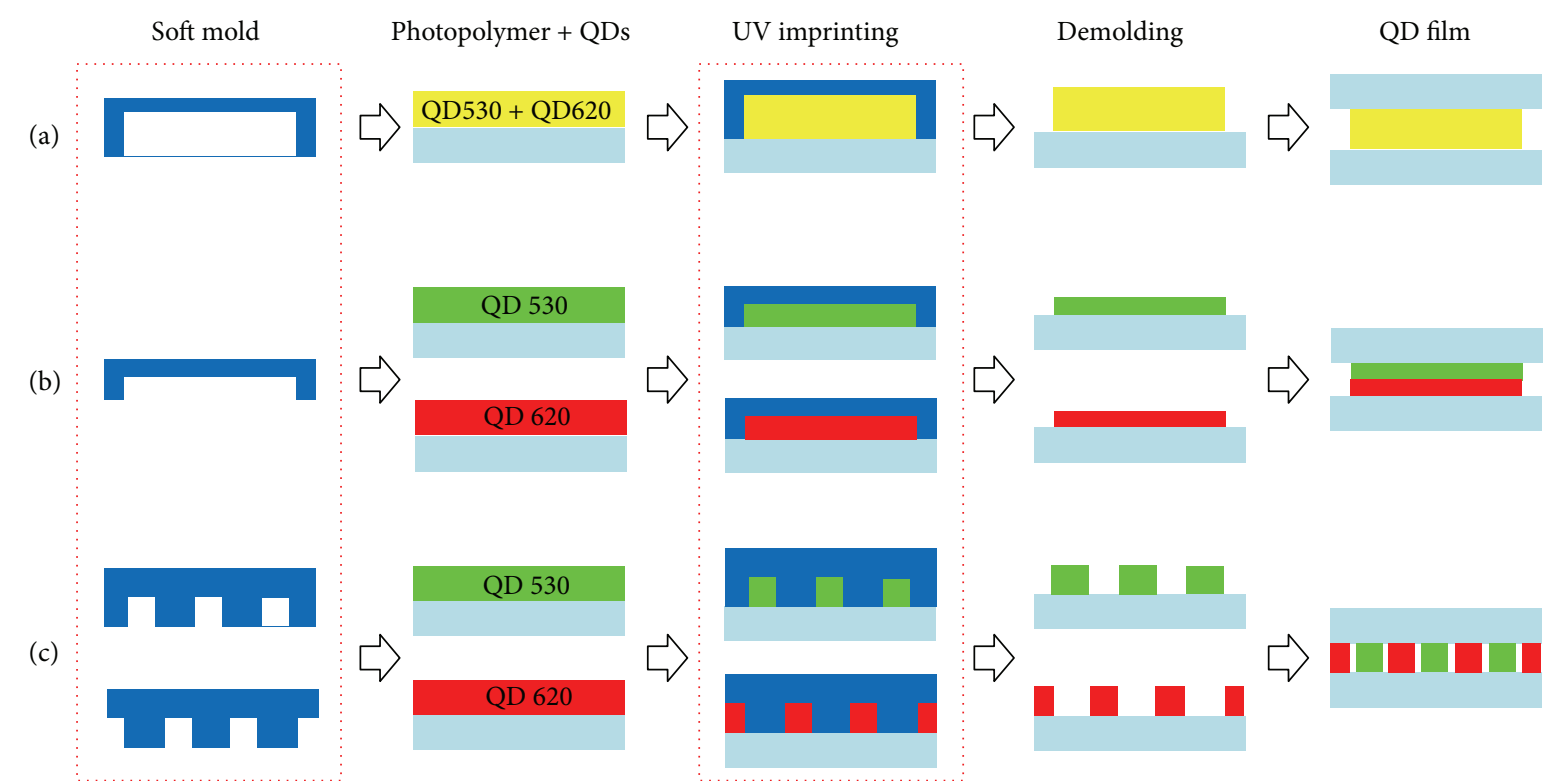

FIGURE 2: Schematic diagram for the fabrication of QD phosphor films by a UV imprinting process for (a) mixed, (b) separately layered, and (c) 2D island-pattern structure.

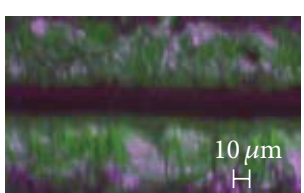

(a)

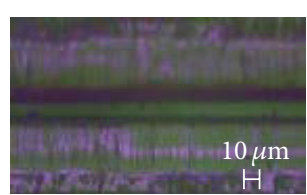

(b)

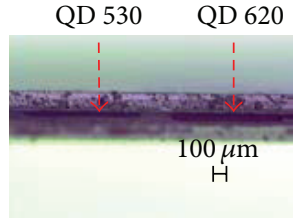

(c)

FIGURE 3: The photomicrographs in the cross-sectional view for QD phosphor films prepared by a UV imprinting process with (a) mixed, (b) separately layered, and (c) 2D island-pattern structure.

and used without any modification. After dispensing in the solvent of toluene, the QD solution was blended with the photopolymer (SM01, Minuta Technology Co., Ltd.) at a concentration of 1 wt.\% of QDs and placed under a vacuum chamber to remove the solvent and undesirable bubbles. Finally, an ultraviolet (UV) imprinting process was applied to produce different structures of QD phosphor films such as the mixed, separately layered, and 2D island-patterns. Figure 2 shows a schematic diagram for the fabrication of QD phosphor films using a UV imprinting process. Soft molds were prepared by a photolithographic process on $\mathrm{Si}$ wafer and the following replicating process to the soft material, as reported before [11]. For Si master, Si wafer was spin coated with CNR-4400-15 photoresist (Advanced Chemtech, Inc., USA), followed by the exposing process with UV light using an MA6 mask aligner (Suss MicroTec, Germany). A developing process was followed to create the engraved area of $23 \times 23 \mathrm{~mm}^{2}$. Then, the UV transparent soft molds were fabricated with a MINS-311 polymer (Minuta Technology Co., Ltd.) by replicating the Si master. In this study, four different soft molds were prepared to realize various QD phosphor film structures. Then, QD coating solution was poured into PET substrate, which was pressed by the soft mold and followed by UV irradiation. For the mixed QD film, two coating solutions were mixed and used for the UV imprinting to produce the film thickness of $25 \mu \mathrm{m}$.

Two soft molds having an engraved form of $12.5 \mu \mathrm{m}$ depth were used to produce two QD films of $530 \mathrm{~nm}$ and $620 \mathrm{~nm}$ QDs and attached together for the separately layered structure (or layer-by-layer (LBL)) of QD film. For 2D islandpatterns of QD film as shown in Figure 2(c), the engraved form of $25 \mu \mathrm{m}$ depth having many island-patterns of $500 \times$ $500 \mu \mathrm{m}^{2}$ inside was used separately to produce the $530 \mathrm{~nm}$ and 620nn QD film. Then two QD films were attached together under the microscopy to align them using the special alignment marks which was included in the Si master. After attaching two QD films, the QD island-patterns with an airgap of $60 \mu \mathrm{m}$ between two different size QD islands were realized. Figure 3 shows the photomicrographs in the crosssectional view for three different QD phosphor films prepared on PET substrate. All QD films have same thickness of $25 \mu \mathrm{m}$ and same QD concentration of $1 \mathrm{wt} . \%$ for both $530 \mathrm{~nm}$ and $620 \mathrm{~nm}$ QDs.

To understand the effect of reabsorption loss, photoluminescence (PL) intensity spectrum was measured for the fabricated three different QD films using a PL spectrometer (PerkinElmer LS45), as shown in Figure 4. In the mixed structure of QD film, represented by green color in Figure 4, a relatively lower intensity was observed from green QDs while 


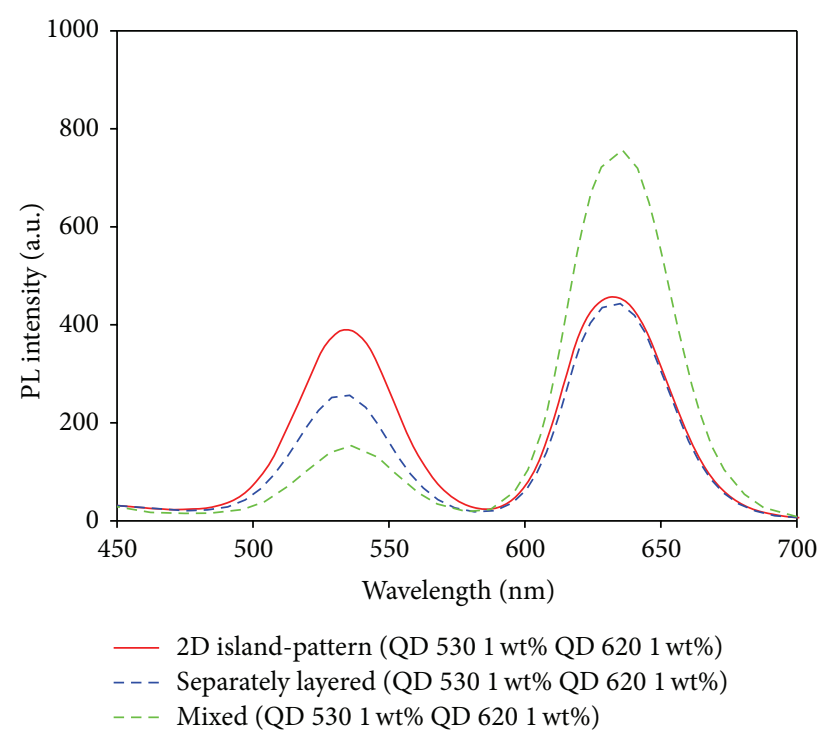

FIGURE 4: The graph of PL intensity measured from the mixed, separately layered, and 2D island-patterns of QD phosphor films.

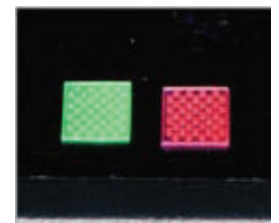

(a)

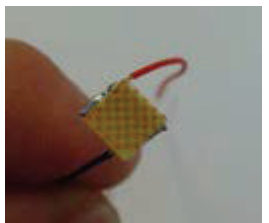

(b)

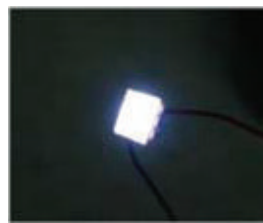

(c)

FIGURE 5: The fabricated white LEDs with QD phosphor films of 2D island-pattern: (a) 2D island-pattern of QD films, (b) white LED after packaging, and (c) white LED with the electrical power on.

a relatively higher intensity occurred by red QDs. This result reveals that there is severe reabsorption at $620 \mathrm{~nm}$ red QDs for the light emitted from $530 \mathrm{~nm}$ green QDs since two QDs were scattered and interfered easily in the mixed structure of QD films. In case of the separately layered structure of QD film, represented by blue color in Figure 4, the $530 \mathrm{~nm}$ green and $620 \mathrm{~nm}$ red peaks show 53\% higher and $40 \%$ lower intensity than those of mixed QD film, respectively. This result means that the separate placement of different QDs in upper and lower layers helps to reduce the reabsorption loss even though there is still some loss in the vertical stacking direction of QD layers. Finally, the PL spectrum from 2D island-patterns of QD films shows a remarkable enhanced intensity for $530 \mathrm{~nm}$ green QDs with the similar intensity from $620 \mathrm{~nm}$ red QDs. Thus we can conclude that 2D island-pattern of QD phosphor films proposed in this study can reduce the reabsorption loss effectively among different size QDs to enhance the optical efficiency in the QD film.

\section{Results and Discussion}

\subsection{White LEDs Based on QD Phosphor Films}

3.1.1. Fabrication and Evaluation of White LEDs. Since more converted green and red lights are required to produce white LEDs, QD concentration was increased to $10 \mathrm{wt} . \%$ of $530 \mathrm{~nm}$ QDs and $5 \mathrm{wt} . \%$ of $620 \mathrm{~nm}$ QDs while the film thickness was kept same as $25 \mu \mathrm{m}$. Three different structures of QD films were fabricated again and diced into small pieces of 5 $\times 5 \mathrm{~mm}^{2}$ to place on top of 5050 blue LED package, as shown in Figure 5(a). InGaN-based blue LED chips (Lumimicro Co., Korea) were used with the peak wavelength of $455 \mathrm{~nm}$ and the optical power of $200 \mathrm{~mW}$. Then silicone epoxy is filled in LED chip package and covered by above diced QD phosphor film as a remote type phosphor. Figure 5 shows the fabricated white LEDs with 2D island-pattern of QD films.

Then, the QD based white LEDs were evaluated using an integrating sphere to obtain color coordinates as well as intensity spectrum. Figure 6 shows the intensity spectrum for QD based white LEDs prepared with three different structures of QD phosphor films. As expected, there is relatively strong peak from $530 \mathrm{~nm}$ green QDs for white LEDs prepared with $2 \mathrm{D}$ island-pattern structure due to less reabsorption by $620 \mathrm{~nm}$ red QDs. In addition, white LED prepared with the mixed structure resulted in relatively lower peak of $530 \mathrm{~nm}$ and relatively higher peak of $620 \mathrm{~nm}$. To evaluate the relative QD conversion efficiency quantitatively in the QD phosphor film, we define the color conversion efficiency as

$$
\begin{aligned}
& \text { color conversion efficiency }(\%) \\
& =\frac{\text { total converted optical power to green and red }}{\text { the used optical power of blue light }} \\
& \quad \times 100 \text {. }
\end{aligned}
$$




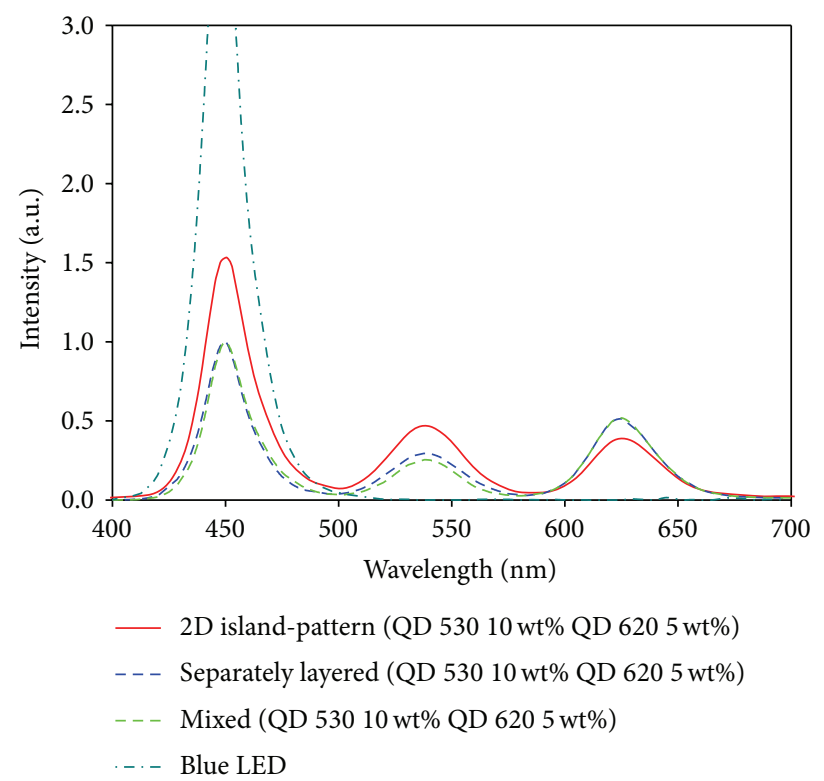

FIGURE 6: Intensity spectrum for white LEDs prepared with different structures of QD phosphor films including the mixed, separately layered, and 2D island-pattern of QD phosphor film.

TABLE 1: Measurement data on optical power for white LEDs prepared with the mixed, separately layered, and 2D island-patterns of QD film.

\begin{tabular}{lccc}
\hline & & Input optical power of blue LED (200 mW) \\
& Mixed & Separately layered & 2D island-pattern \\
\hline Total optical power of LED (mW) & 67.909 & 69.408 & 81.453 \\
Blue & 21.772 & 22.164 & 32.225 \\
Green (QD 530) & 8.886 & 12.632 & 14.614 \\
Red (QD 620) & 37.251 & 34.612 & 34.614 \\
\hline Color conversion efficiency (\%) & 25.89 & 26.57 & 29.34 \\
\hline
\end{tabular}

Since the penetrated amount of blue light is different from three different QD film structures, we considered the used (or decreased) power of blue light as a reference for the calculation of color conversion efficiency. The measured optical power is summarized with relative intensity of blue, green, and red lights in Table 1. White LEDs prepared from the mixed and separately layered (or LBL) structures show similar values of color conversion efficiency with $25.89 \%$ and $26.57 \%$, respectively. In case of white LED prepared with $2 \mathrm{D}$ island-pattern structure, it was improved effectively with $29.34 \%$ which means $13.3 \%$ and $10.4 \%$ higher than those prepared with the mixed and separately layered structure, respectively. The color coordinates for white LEDs prepared by the mixed, separately layered (or LBL), and 2D islandpattern were also measured as $(0.2883,0.2028),(0.2598$, $0.2084)$, and $(0.2602,0.2472)$. Thus it is also clear from the color coordinates that the reabsorption loss among QDs can be reduced to present more green light in white LEDs prepared with 2D island-patterns of QD film.

3.1.2. Simulation on the Air-Gap between QD Islands. To verify the effectiveness of air-gap between QD islands in the 2D island-pattern, 2D continuous pattern without airgap was also evaluated by optical simulation. The simulation based on the ray tracing and Monte Carlo method was performed which is reported in our previous paper [10]. White LED includes blue LED chips of $455 \mathrm{~nm}$ wavelength with Lambertian distribution and $200 \mathrm{~mW}$ optical power at the operation current of $60 \mathrm{~mA}$. LED package and remote type QD phosphor films are kept same as those in case of experimental measurement, including the film thickness of $25 \mu \mathrm{m}$ and the QD concentration to $10 \mathrm{wt} . \%$ of $530 \mathrm{~nm}$ QDs and $5 \mathrm{wt} \%$ of $620 \mathrm{~nm}$ QDs. An optical reflectance on the surface of LED package was assumed to be $85 \%$ with a Lambertian distributed scattering. Absorption and emission spectrum as well as quantum efficiency of QDs in the QD film were measured experimentally and used in this simulation. The quantum efficiency of $530 \mathrm{~nm}$ and $620 \mathrm{~nm}$ QDs in the film was 0.6 and 0.5 , respectively. For the comparison, white LEDs prepared with different structures of QD films such as mixed and separately layered QD film were also evaluated. The quantitative evaluation data on the color conversion efficiency as a function of QD film structures are summarized in Table 2. The color conversion efficiency of white LEDs prepared with 2D island-pattern is best with $29.8 \%$ which is very similar to that of experimental result. In case of white LED prepared with 2D continuous pattern, almost the same value to that of the separately layered QD structure is shown. 
TABLE 2: Simulation results for white LEDs prepared with mixed, separately layered, 2D island-pattern, and 2D continuous pattern of QD films.

\begin{tabular}{lcccc}
\hline & \multicolumn{3}{c}{ Input optical power of blue LED (200 mW) } \\
& Mixed & Separately layered & 2D island-pattern & 2D continuous pattern \\
\hline Total optical power of LED $(\mathrm{mW})$ & 64.682 & 68.266 & 98.340 & 72.903 \\
Blue & 19.875 & 20.116 & 55.168 & 24.628 \\
Green (QD 530) & 10.000 & 9.170 & 17.255 & 18.198 \\
Red (QD 620) & 34.806 & 38.980 & 25.917 & 30.077 \\
\hline Color conversion efficiency (\%) & 24.87 & 26.77 & 29.81 & 27.53 \\
\hline
\end{tabular}

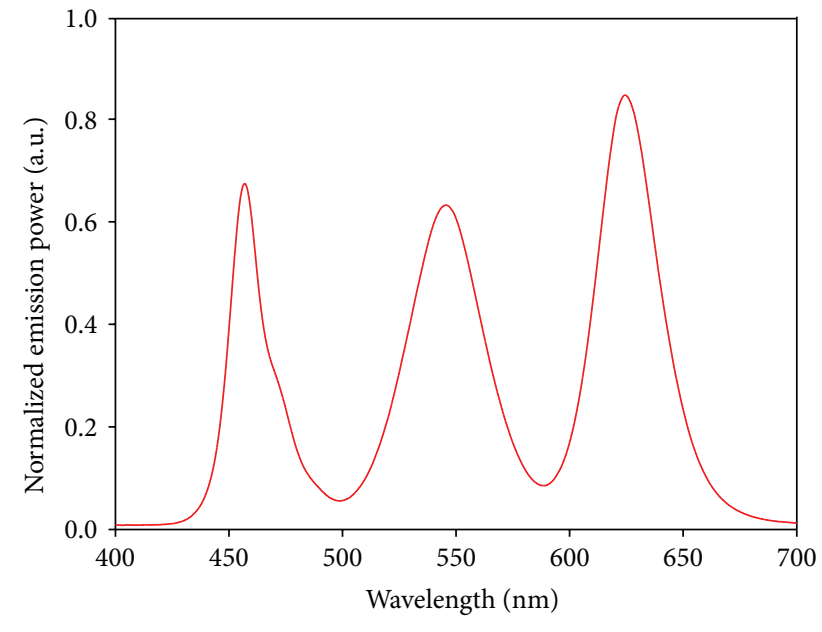

— 2D island-pattern (QD $53025 \mathrm{wt} \%$ QD $62010 \mathrm{wt} \%$ )

(a)

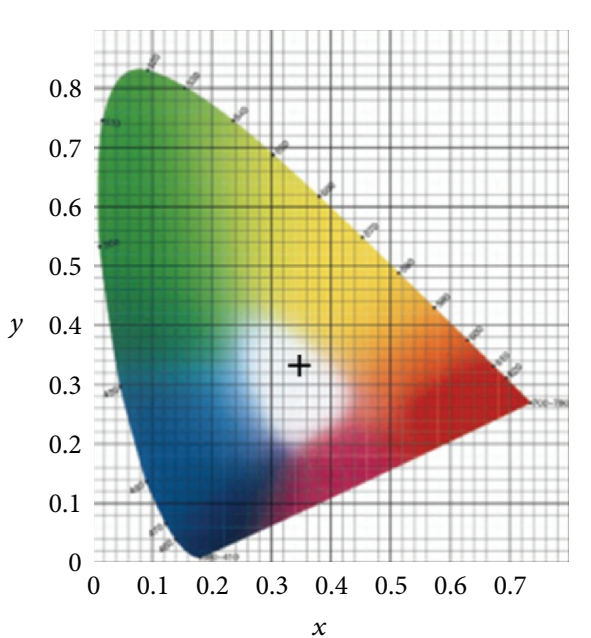

(b)

FIGURE 7: (a) Intensity spectrum and (b) color coordinates for high luminous white LED prepared with 2D island-pattern of QD phosphor film, resulting in the high luminous efficiency of $62.2 \mathrm{~lm} / \mathrm{W}$ and CRI of 83 at the operation current of $60 \mathrm{~mA}$.

This similarity is related to the fact that there is an optical interference laterally at the interface between $2 \mathrm{D}$ patterns of different size QDs to produce a reabsorption loss at the interface in case of $2 \mathrm{D}$ continuous pattern film. In the separately layered structure, there is an optical interference vertically between upper and lower layers. Thus, it is understood that $2 \mathrm{D}$ island-pattern having air-gap between QD islands is the best structure in the QD phosphor film.

3.2. High Luminescence White LEDs. Finally, high luminescence white LED with relatively high CRI in general CCT range was fabricated and evaluated. Figure 7 shows intensity spectrum for white LED prepared with 2D island-pattern of QD dispersed photopolymer film. To realize this white LED, the concentration of QDs was increased to $25 \mathrm{wt} . \%$ of $530 \mathrm{~nm}$ QDs and $10 \mathrm{wt} . \%$ of $620 \mathrm{~nm}$ QDs while the film thickness was kept same as $25 \mu \mathrm{m}$. The white LED developed in this study shows high luminous efficiency of $62.2 \mathrm{~lm} / \mathrm{W}$ at the operation current of $60 \mathrm{~mA}$ and high CRI of 83 with the color coordinates of $(0.3585,0.3572)$ and CCT of $4537 \mathrm{~K}$. Actually, we believe that these experimental data for the luminous efficiency and CRI from the QD based white LED are a leading edge result in this field. Thus, we can conclude that 2D island-pattern of QD phosphor films has an advantage to realize high luminous efficiency as well as high CRI.

\section{Conclusions}

To overcome the reabsorption loss among different size QDs in the QD based white LEDs, new structure of QD phosphor films was proposed and fabricated to enhance the luminous efficiency as well as color conversion efficiency. New QD phosphor film consists of 2D island-pattern of different size QDs in a zigzag form with a small air-gap such as $60 \mu \mathrm{m}$ between QD islands. The air-gap helps to control the optical path at the interface to reduce the lateral reabsorption loss, resulting in the improvement of optical efficiency of white LEDs. Different structures were fabricated with same concentrations and thicknesses using a UV imprinting process, including the mixed, separately layered, and 2D islandpattern of QD phosphor films.

A comparative analysis was performed both in simulation and experiment to confirm the enhancement of optical efficiency due to the reduced reabsorption loss among QDs in the 2D island-pattern of QD film. The color conversion efficiency shows the improvement of $10.4 \%$ and $13.3 \%$, compared to those of the separately layered and mixed structures, respectively. The effectiveness of air-gap in newly proposed QD film structure was also verified by simulation after the comparison with 2D continuous QD pattern without air-gap. Finally, white LEDs were fabricated with $2 \mathrm{D}$ island-pattern 
of QD phosphor film to realize high luminous efficiency of $62.2 \mathrm{~lm} / \mathrm{W}$ and high CRI of 83 with color coordinates of (0.3585 and 0.3572$)$ CCT of $4537 \mathrm{~K}$. Thus, we can conclude that $2 \mathrm{D}$ island-pattern of $\mathrm{QD}$ phosphor films is the best structure to realize high luminescence white LEDs.

\section{Conflict of Interests}

The authors declare that there is no conflict of interests regarding the publication of this paper.

\section{Acknowledgments}

This work was supported by the Industrial Strategic Technology Development Program (no. 10035274) and the Advanced Technology Center Program (no. 10042178) funded by the Ministry of Trade, Industry and Energy of Korea.

\section{References}

[1] S. Coe, W.-K. Woo, M. Bawendi, and V. Bulović, "Electroluminescence from single monolayers of nanocrystals in molecular organic devices," Nature, vol. 420, no. 6917, pp. 800-803, 2002.

[2] V. L. Colvin, M. C. Schlamp, and A. P. Alivisatos, "Light-emitting diodes made from cadmium selenide nanocrystals and a semiconducting polymer," Nature, vol. 370 , no. 6488 , pp. 354357, 1994.

[3] H.-S. Chen, C.-K. Hsu, and H.-Y. Hong, "InGaN-CdSe-ZnSe quantum dots white LEDs," IEEE Photonics Technology Letters, vol. 18, no. 1, pp. 193-195, 2006.

[4] R. A. J. Janssen and J. W. Stouwdam, "Red, green, and blue quantum dot LEDs with solution processable $\mathrm{ZnO}$ nanocrystal electron injection layers," Journal of Materials Chemistry, vol. 18, no. 16, pp. 1889-1894, 2008.

[5] D. J. Norris, M. G. Bawendi, and L. E. Brus, Molecular Electronics: A Chemistry for the 21st Century, Monograph Chapter 9, Blackwell Science, 1997.

[6] S. Nizamoglu, T. Ozel, E. Sari, and H. V. Demir, "White light generation using $\mathrm{CdSe} / \mathrm{ZnS}$ core-shell nanocrystals hybridized with InGaN/GaN light emitting diodes," Nanotechnology, vol. 18, no. 6, Article ID 065709, 2007.

[7] W. Chung, K. Park, H. J. Yu, J. Kim, B.-H. Chun, and S. H. Kim, "White emission using mixtures of CdSe quantum dots and PMMA as a phosphor," Optical Materials, vol. 32, no. 4, pp. 515-521, 2010.

[8] S. Nizamoglu and H. V. Demir, "Hybrid white light sources based on layer-by-layer assembly of nanocrystals on near-UV emitting diodes," Nanotechnology, vol. 18, no. 40, Article ID 405702, 2007.

[9] S. U. Ishii, S. Nakanishi, Y. Yoshida et al., "Fabrication of a quantum dot-polymer matrix by layer-by-layer conjugation," Journal of Photochemistry and Photobiology A: Chemistry, vol. 183, no. 3, pp. 285-291, 2006.

[10] M. Shin, H. Hong, H. Kim, and Y. Kim, "Enhancement of optical extraction efficiency in white LED package with quantum dot phosphors and air-gap structure," Applied Physics Express, vol. 7, no. 5, Article ID 052101, 2014.

[11] J. Lim, H.-G. Hong, J. Han et al., "Design and fabrication of a metallic nanostamp using UV nanoimprinting and electroforming for replicating discrete track media with feature size of $35 \mathrm{~nm}$," Journal of Vacuum Science and Technology B: Microelectronics and Nanometer Structures, vol. 31, Article ID 011801, 2013. 

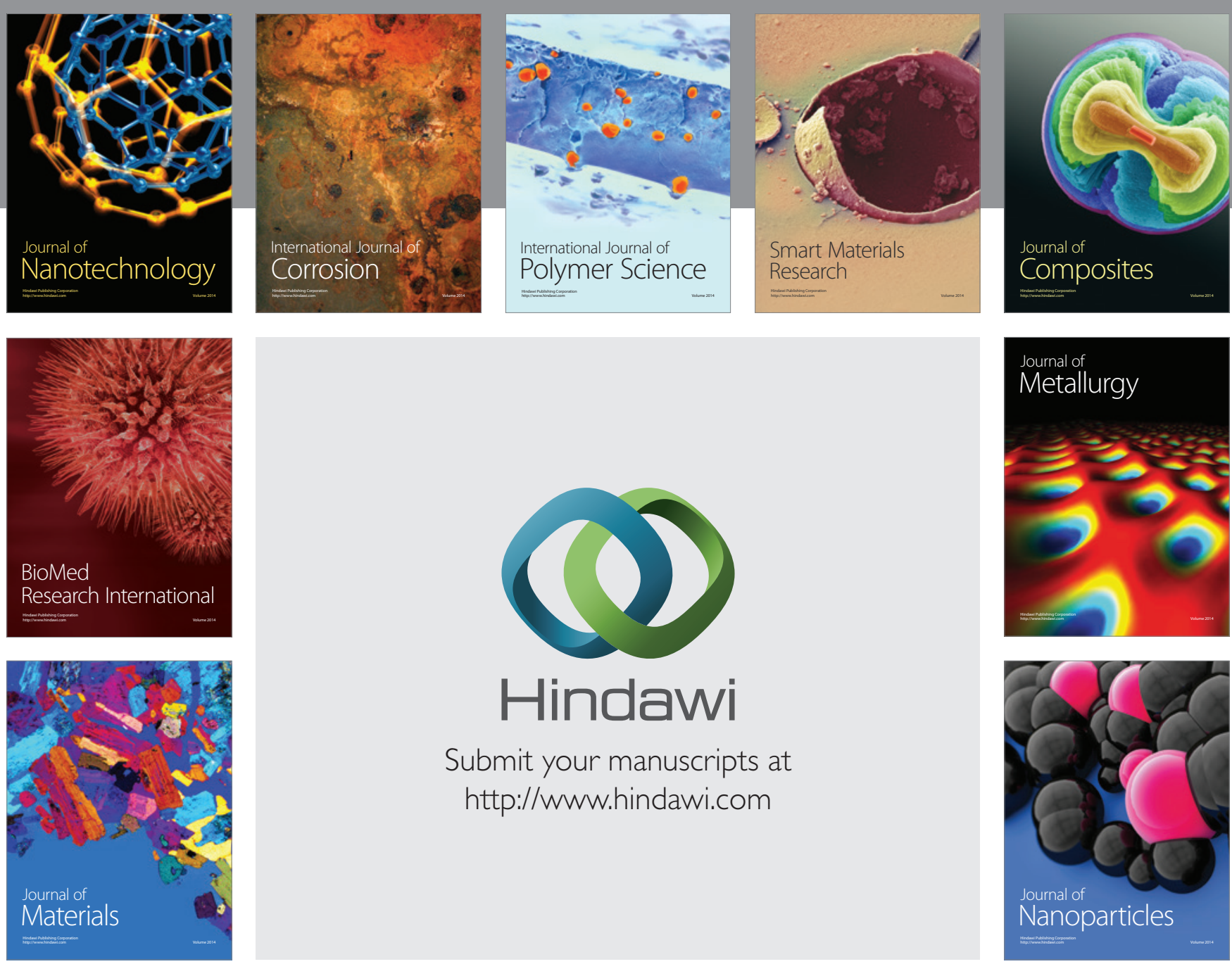

Submit your manuscripts at http://www.hindawi.com
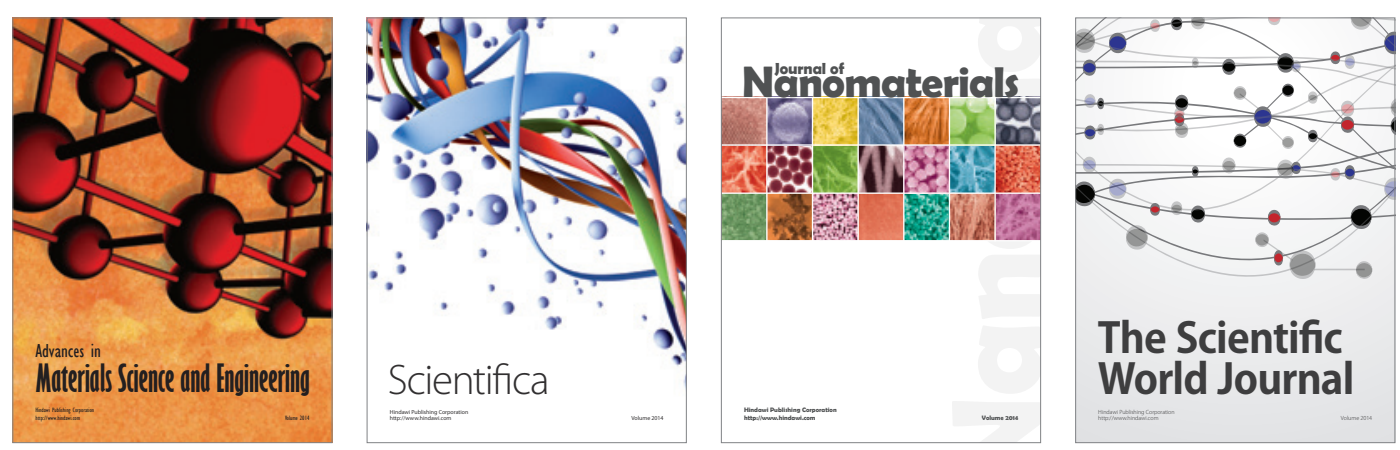

\section{The Scientific World Journal}
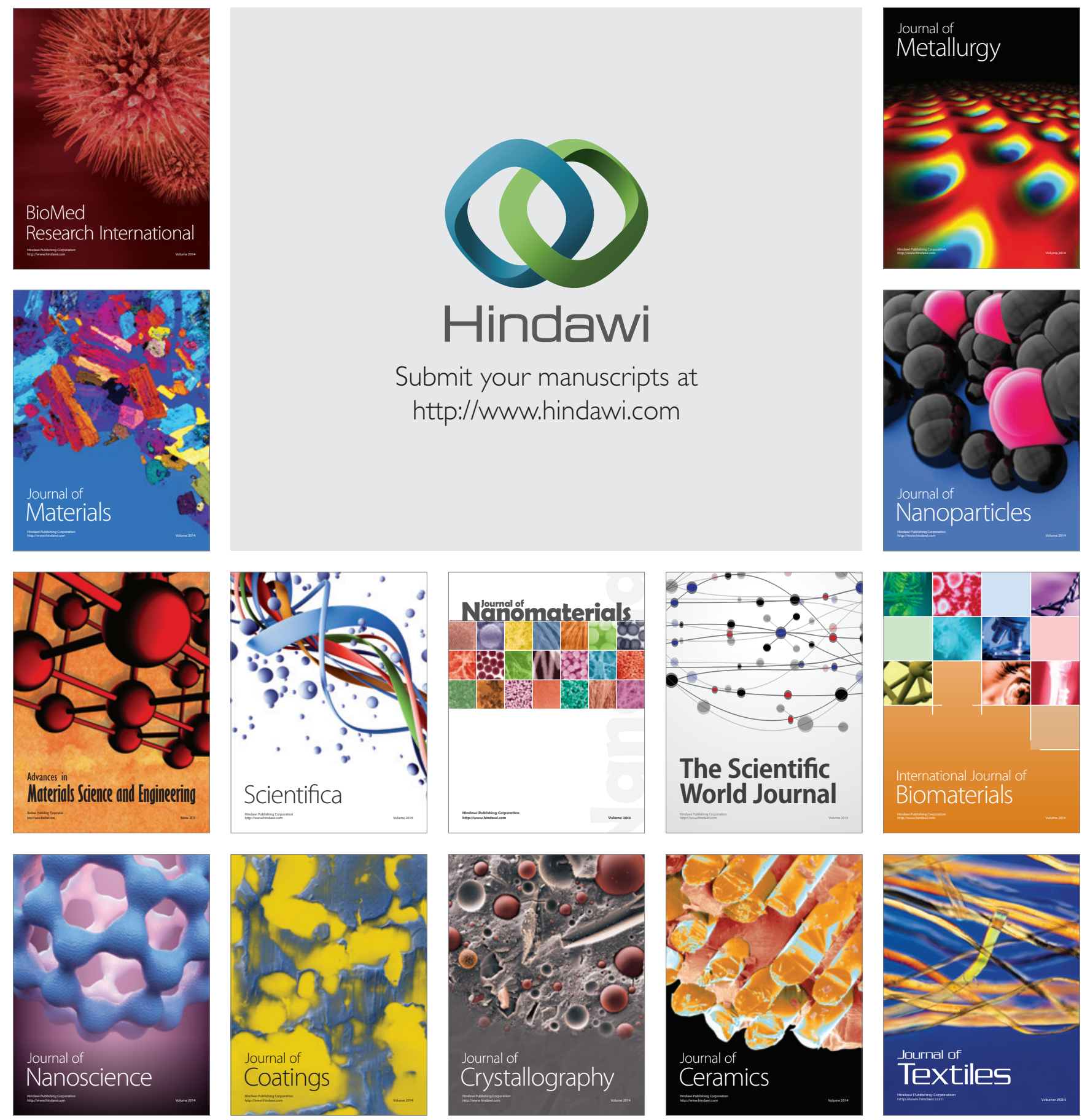